\title{
Protocol for a systematic review of randomized trials of knee arthroplasty decision aids and shared decision-making approaches
}

\author{
Daniel L. Riddle ${ }^{1 *}$ (D) Trisha Sando², Talicia Tarver ${ }^{2}$, James Slover ${ }^{3}$, Robert A. Perera ${ }^{2}$, Rafael Sierra ${ }^{4}$,
} Juan P. Brito ${ }^{4}$ and Victor M. Montori ${ }^{4}$

\begin{abstract}
Background: Shared decision-making is an approach to making treatment-based decisions that rely on the patient encounter and clear discussions between the patient and the healthcare provider. Patients with arthritis of the knee frequently seek care, and depending on arthritis severity and impact on daily life, joint arthroplasty may be considered as a treatment option. We will conduct a systematic review of shared decision-making trials in knee arthroplasty to determine the types of shared decision-making approaches used and their impact on care received.

Methods: Our systematic review will describe and critically appraise shared decision approaches used in randomized trials of patients undergoing knee arthroplasty, the types of outcomes reported, and the impact of these approaches on the patients' care. We will use the following databases: PubMed, Web of Science, Embase, CINAHL, PsycINFO, and the Cochrane Library, from inception through December 2018. Additionally, we will assess ongoing research by querying experts and searching trial registries.

Discussion: This study will characterize shared decision-making (SDM) approaches in knee arthroplasty randomized clinical trials and will summarize their effects of SDM on clinical and patient-reported outcomes. We anticipate this review will bring to light knowledge gaps and inform further research into the design and use of shared decisionmaking approaches in lower extremity arthroplasty.
\end{abstract}

Systematic review registration: PROSPERO CRD42019123586

Keywords: Knee, Arthroplasty, Shared decision-making

\section{Background}

Total knee arthroplasty (TKA) is the most common major surgical procedure conducted in the USA. Approximately one million persons underwent TKA in the USA in 2015, double the volume as compared to 2005 [1]. Demand is likely to continue to increase given that the population of middle-aged and older persons is increasing [2] and, on average, gaining weight [3].

\footnotetext{
* Correspondence: dlriddle@vcu.edu

'Departments of Physical Therapy, Orthopaedic Surgery and Rheumatology, West Hospital, Virginia Commonwealth University, Room B100, 1200 East Broad Street, Richmond, VA 23298-0224, USA

Full list of author information is available at the end of the article
}

Patient selection for TKA is driven primarily by the surgeon's assessments of a patient's knee arthritis severity, pain intensity, and related limitations in addition to surgical fitness as well as the patient's willingness to undergo the procedure. The Centers for Medicare and Medicaid Services (CMS) has recently mandated a shared decision-making (SDM) approach for some cardiovascular and cancer procedures [4], and these mandates could eventually be applied to other procedures including TKA. Professional organizations like the American Academy of Orthopaedic Surgeons (AAOS) endorse a SDM approach for patients being considered for TKA. The AAOS position statement entitled Shared Physician-Patient Responsibilities [5] reads, "The orthopaedic surgeon should engage in informed shared

(c) The Author(s). 2019 Open Access This article is distributed under the terms of the Creative Commons Attribution 4.0 International License (http://creativecommons.org/licenses/by/4.0/), which permits unrestricted use, distribution, and 
decision making with the patient using the patient's values and respect the patient's decision even if it is in disagreement with the physician's recommendation." The AAOS does not define procedures for applying SDM to clinical practice.

An SDM interaction between a patient and a healthcare provider has been defined as having six key elements [6]. The first element is labeled "situation diagnosis" and requires the clinician to understand the patient's health-related situation and identify aspects of the situation that require action. The second element, "choice awareness," involves making the patient aware of the various options to address the health-related situation while clarifying the importance of patient preference. The conversation then emphasizes the various options (i.e., "option clarification") and how these options and their associated benefits and harms align with patient preferences (i.e., "discussion of harms and benefits"). The patient and provider are then in a position to discuss how the various options fit with the patient's preferences (i.e., "deliberation of patient preferences"). In the final step (i.e., "making the decision"), the patient and clinician together reach a decision for managing the health-related situation. The three-talk model for SDM [7] includes a very similar sequence of elements as described above. Systematic reviews of randomized trial evidence suggest that the impact of SDM on patient cognitive outcomes such as knowledge, affect, and attitude is generally enhanced and decisional conflict is reduced relative to usual care. Health and behavioral outcomes such as self-rated health and biological measures are generally less influenced by SDM and vary depending on the study $[8,9]$.

Decision aids are tools that assist in some aspects of the shared decision-making process. A decision aid (DA) usually contains content of relevance to a particular clinical decision but typically is delivered to the patient prior to the encounter with the clinician and does not include additional elements of SDM that occur during the encounter. In particular, DAs do not typically extend beyond content related to the disorder or treatments of interest and potential benefits and harms related to a healthcare choice for the disorder of interest. For example, in the TKA literature, Bozic and colleagues used a DA developed by the Informed Medical Decision Foundation to determine if the use of the DA improved patients' knowledge regarding hip or knee osteoarthritis and their stage of decision-making [10]. Patients randomly assigned to the DA arm were given a digital video disc (DVD) and booklet prior to their surgeon visit. The DVD and booklet described knee OA natural history as well as content describing risks and benefits of surgical and nonsurgical options for osteoarthritis. Additionally, patients had a phone-based conversation with a health coach to prepare a list of questions for the surgeon. The DA did not include content related to whether or how the surgeon considered patient preferences when making a TKA recommendation or guidance for either the patient or surgeon regarding the conversation during the encounter. DAs also have been shown to impact cognitive outcomes such as knowledge or decisional conflict relative to usual care [11].

We found no systematic reviews of the TKA DA/SDM literature that specifically determined the types of DA/ SDM approaches studied, their relationship to key elements of SDM, or the extent to which DA/SDMs actually impacted the care that the patients received. The purposes of this protocol are to (1) identify and critically appraise the TKA randomized trial literature that has examined the impact of DA/SDM approaches on TKA decisions and outcomes, (2) assess the extent to which the TKA trials of DA/SDM approaches incorporated SDM elements, (3) determine the types of outcomes that were studied and the effects of DA/SDM approaches on these outcomes relative to alternative interventions, and (4) determine the extent to which the DA/ SDM approaches used in the trials impacted the care that the patients received. This protocol was modeled after a recently published protocol by Wieringa and colleagues [6].

\section{Methods}

Study design

Our protocol design was based on the Preferred Reporting Items for Systematic Review and Meta-analysis Protocols (PRISMA-P) [12].

\section{Study type}

Randomized clinical trials will be selected if they compare the use of DA/SDM approaches to either usual care or an active control intervention. No time limit will be established for the search, and all potential study or clinical settings will be included.

\section{Eligibility criteria}

All studies enrolling patients with arthritis and who were scheduled to undergo TKA will be included. No restrictions will be placed on the type of arthritis being treated (e.g., osteoarthritis, rheumatoid arthritis, post traumatic arthritis). Studies that include a mixed sample of patients undergoing either TKA or total hip replacement (THA) will be included only if subgroup data and analyses are provided for patients undergoing TKA. Approximately 1 million patients undergo TKA each year in the USA [1], and a substantial number of TKAs are conducted worldwide. Additionally, the outcomes, recovery trajectories, and patient perceptions of recovery are different for TKA versus THA, and therefore, the focus of this systematic review is on TKA. 


\section{Interventions and comparators of interest}

All studies that randomize patients who plan to undergo TKA to a DA/SDM treatment arm and a usual care or active control arm will be included. No restrictions will be placed on the type of usual care or active control that is provided. There also will be no restriction on the type of TKA surgical procedure or the experience of the surgeon conducting the surgery. Patients undergoing either TKA or unicompartmental knee arthroplasty will be included. Data will be disaggregated for TKA and unicompartmental arthroplasty if reported by the authors.

All interventions will be described including those that aim to support elements of SDM during the encounter as well as DAs used before or during the encounter. Descriptions will summarize the key elements of the SDM approach or DA and any associated interventions such as the use of a health coach to aid in DA content understanding [13]. Alternative interventions may also include general educational content about arthritis or usual care treatment and these will be described. Content included in the DA or SDM approach will be specified. For example, Hawker and colleagues used a DA consisting of a 50-min video and booklets that summarized surgical and non-surgical treatment options and their associated benefits, risks, and probabilities [14].

\section{Information sources and search strategy}

The plan was based on a comprehensive approach to RCT identification using the following databases (and corresponding database platforms): MEDLINE (PubMed), Web of Science (Web of Science Core Collection), Embase (Ovid), CINAHL (EBSCO), PsycINFO (APA PsycNET), and the Cochrane Library (CENTRAL). The search strategy was developed with the assistance of a research librarian (TT). All databases will be searched from inception through December 2018. No restrictions will be based on language. Foreign language RCTs will be translated using Google Translate. Reference lists of included studies will be checked for additional sources. All authors of the papers selected for review will be contacted via email to assess whether additional research is ongoing. Finally, several electronic databases will be reviewed to assess for the presence of ongoing RCTs of DA/SDM approaches in TKA. These databases will include the following: ISRCTN.org, Clinicaltrials.gov, and PROSPERO registry. If multiple articles are published from a single RCT, all will be included to assure coverage of all interim analysis time points. Searches of all databases will be documented in a table to assure complete reporting of all search results. The search strategy and preliminary number of papers meeting the search strategy for each database are presented in Additional file 1.

\section{Study records}

A searching data management software (i.e., Covidence) will be used by two independent reviewers (DLR and TS) to identify and track searches from all relevant databases. The two reviewers will screen all titles and abstracts independently identified from the search strategy for the included databases. Full reports will be obtained for each title/abstract judged to be potentially relevant. Reasons for exclusion will be reported for each database using the PRISMA flow diagram. A third independent reviewer (JS) will resolve any discrepancies resulting from the initial review. The extent of agreement for excluded papers and papers selected for inclusion by the two reviewers will be assessed using the Kappa statistic $(K)$, an agreement index that accounts for chance agreement [15].

A standardized data extraction table will be used to collect relevant data on each included trial (see Additional file 2). The table was modified based on the recently published data extraction table by Wieringa and colleagues [6]. The data table includes key headings of the publication details, study design, population of interest, sociodemographic characteristics of the samples, inclusion and exclusion criteria, study setting, details of experimental intervention and comparison intervention, duration of follow-up and outcomes studied, and extent of effectiveness for both cognitive and clinical outcomes of the DA/SDM approach in relation to the comparator. In addition, a summary of the six key elements of SDM tools [6] will be collected for each RCT. Particular attention will be paid to the current availability of DA/SDM approaches used in the included studies. If the DA/SDM is commercially available, this will be reported. We will contact the corresponding author of all eligible studies with three purposes: (a) to verify that we have characterized their studies correctly, (b) to complete any missing data, and (c) to ask for any unpublished studies. The method of contact will be via email, sending two email notices separated by 2 weeks. If there is no answer, we will pursue contact of the first author or senior author (if different from the corresponding one) in similar fashion.

\section{Outcomes and prioritization}

All outcomes collected at every time point will be included in the review. The primary outcome of interest will be cognitive/affective outcomes including but not limited to decisional conflict [16], content knowledge regarding arthritis, and treatment decision preference. Secondary outcomes will be clinical outcomes including but not limited to satisfaction with surgical outcome, patient-reported pain and functional outcome, surgical versus non-surgical treatment decisions made during the encounter, and any other clinical outcome data collected in any of the included studies. Either cognitive/affective outcomes or clinical outcomes may be proximal (e.g., measured immediately after the clinician encounter) or distal (e.g., typically measured weeks or months after the encounter) [17]. 
Outcome measures used in DA studies tend to focus primarily on proximal cognitive/affective outcomes related to whether the patient's review of the DA content prior to the clinician encounter led to improvements in, for example, decisional conflict or content knowledge. Minimal emphasis is typically placed on the measurement of outcomes of the encounter itself. Common outcome tools in DA studies include but are not limited to the decisional conflict scale [18] and the Hip/Knee Osteoarthritis Decision Quality Instrument a scale, an example of a content knowledge survey [19]. In contrast, SDM studies place major focus on the clinical encounter and whether the SDM tool led to clinical decisions and interactions that facilitated and were consistent with patient preferences. Common outcome tools in SDM include but are not limited to the SDM-Q-9 [20] and the OPTION scale [21], both of which are used to collect detailed patient/clinician encounter data. Both DA and SDM approaches have included proximal clinical outcomes including satisfaction with the clinical decision as well as distal clinical outcomes such as 30-day post-treatment adverse event rates [22]. Our review will comprehensively assess the outcomes used in the trials and report the extent to which outcomes assess both cognitive/affective outcomes and clinical outcomes at proximal and distal time points as well as the extent to which the outcomes assess the encounter itself and the decision-making process used during the encounter.

\section{Handling missing data}

If data reported in a study is unclear or missing, we will request information from the authors using the approach described above. All attempted contacts will be documented.

\section{Risk of bias in individual studies}

Bias risk for each trial will be assessed by two independent reviewers (DLR and TS) using the risk of bias tool (RoB 2) developed by the Cochrane Collaboration [17]. This bias instrument assesses five domains of potential bias: the randomization process, deviations from intended interventions, outcome data missingness, outcome measurement, and reported result biases. Each domain is assessed using multiple items, and an overall risk of bias is rated as low, high, or some concerns. The Kappa statistic will be used to judge the extent of agreement for each of the six domains, after accounting for chance agreement, by the two raters. If agreement is less than moderate for a domain-specific risk of bias score (i.e., $K=0.4$ or less) [15], a third reviewer (JS) will arbitrate differences until an acceptable level of agreement is achieved.

\section{Synthesis of study data}

We will describe the DA/SDM approaches for each trial in our review as well as the extent to which each trial addressed the 6 key elements of SDM [6] along with cognitive and clinical outcomes of each trial. Additionally, for each trial, we will report the extent to which the DA/SDM arm impacted the actual care that the patients received, as compared to the usual care/active control arm. For continuous outcomes (e.g., decisional conflict scale [16]), mean differences or mean changes between $\mathrm{AD} / \mathrm{SDM}$ and usual care/active control group, together with $p$ values and 95\% confidence intervals (95\% CIs), will be extracted. For dichotomous outcomes, risk ratios (RRs) or odds ratios (ORs) with 95\% CIs will be extracted or calculated if possible. To display heterogeneity and effect sizes, forest plots will be used. A random effects meta-analysis model to account for both betweenand within-study variance will be used. The pooled effect size across studies will be estimated using a random effects meta-analysis model. Studies will be weighted by the inverse of the variance of the parameter estimates. The $Q$ statistic and $I^{2}$ index will be used to assess heterogeneity across studies. Because we expect substantial inconsistency in participants, interventions, comparators, and outcomes, where sensible, we will conduct a random effects meta-regression with the intent of exploring for subgroup interactions between patient DA and conversational SDM approaches. The "metafor" package in the $\mathrm{R}$ statistical software [18] will be used to complete all analyses with an alpha of 0.05 [19]. We estimate that all data analyses will be completed by the end of October of 2019. Narrative synthesis may be utilized for those content areas in which only one or very few RCTs have been conducted, for example, trials conducted on persons with unicompartmental knee arthroplasty [21] or in TKA access studies of participants who are African American [22].

Protocol amendments prior to the start of data collection will be made by posting changes to the PROSPERO registry information (see CRD42019123586). Changes made after the start of data collection will be reported in subsequent systematic review publications.

\section{Meta-biases}

To examine potential publication bias, a contourenhanced funnel plot will be reported [20].

\section{Confidence in cumulative evidence}

The Grading of Recommendations Assessment, Development and Evaluation (GRADE) approach to judging overall quality of evidence and recommendations as defined in the GRADE Handbook will be applied to the evidence generated in this systematic review [23]. These recommendations include an overall quality of evidence rating for each outcome as high, moderate, low, or very low. The GRADEpro GDT software will be used to generate GRADE data [24]. 


\section{Discussion}

This review will provide a comprehensive summary of the clinical trial evidence related to the use of DA/SDM approaches as applied to patients undergoing TKA, the most common major surgical procedure conducted in the USA. To date, several DA/SDM clinical trials are known to have been published [14, 22, 25-28], and it is likely that the comprehensive search planned as part of the systematic review will identify other trials. The findings from this systematic review will inform future research of DA/SDM approaches for patients with TKA at a time when substantial emphasis is being placed on SDM approaches for beneficiaries by third-party payers such as the Centers for Medicare and Medicaid Services [4]. Specifically, this review will inform the extent to which the current DA/SDM conforms to the six key elements of SDM and the extent to which the studies used DA/SDM methods to guide and influence surgeon/patient conversations regarding TKA candidacy. These findings have potential to not only impact future research on SDM methods related to TKA decisionmaking but may also inform orthopedic surgeons of the practical issues to consider when attempting to incorporate SDM applications in daily practice. Uptake of DA/ SDM approaches in daily practice has been limited and has been attributed to a variety of factors including the perception by orthopedic surgeons that patient outcomes were already optimal, a lack of alternatives to TKA, and concerns regarding medico-legal implications of using a DA/SDM tool [29]. Additionally, while some surgeons may be concerned about the additional time and methods required to incorporate SDM approaches to daily care, evidence suggests that the time necessary to incorporate effective contemporary SDM approaches adds only a few minutes to an encounter [30].

There are limitations to our review. Our focus is on RCTs of DA/SDM approaches for TKA given that RCTs are considered the most valid method for determining causal associations between treatment and outcome [31]. However, we may exclude potentially well-described and well-studied DA/SDM approaches applied only in observational studies. Additionally, our focus is on TKA and the results will not directly apply to DA/SDM approaches applied to other orthopedic surgical approaches though the concepts discussed in the review may assist others in applying DA/SDM principles to other surgical approaches in orthopedics including hip arthroplasty and fracture treatment.

\section{Additional file}

Additional file 1: The search strategy and preliminary number of papers 414 meeting the search strategy for each database. (DOCX $18 \mathrm{~kb}$ )
Additional file 2: A standardized data extraction table used to collect 415 relevant data on each included trial. (DOCX $26 \mathrm{~kb}$ )

\section{Abbreviations}

AAOS: American Academy of Orthopaedic Surgeons; CMS: Centers for Medicare and Medicaid Services; DA: Decision aid; DVD: Digital video disc; PRISMA-P: Preferred Reporting Items for Systematic Review and Meta-analysis Protocols; SDM: Shared decision-making; THA: Total hip arthroplasty; TKA: Total knee arthroplasty; K: Kappa statistic

\section{Acknowledgements}

Not applicable.

\section{Authors' contributions}

DLR designed and wrote the protocol. DLR, TS, TT, JS, RS, JPB, and VMM made substantial contributions and revisions to it. $T$ designed the search strategy, based on a prior systematic review protocol [6]. The final version of this protocol was reviewed and approved by all authors.

\section{Funding}

There was no funding for this study.

Availability of data and materials Not applicable.

Ethics approval and consent to participate Not applicable.

\section{Consent for publication}

Not applicable.

\section{Competing interests}

The authors declare that they have no competing interests.

\section{Author details}

'Departments of Physical Therapy, Orthopaedic Surgery and Rheumatology, West Hospital, Virginia Commonwealth University, Room B100, 1200 East Broad Street, Richmond, VA 23298-0224, USA. ${ }^{2}$ Virginia Commonwealth University, Richmond, VA, USA. ${ }^{3}$ New York University, Langone Medical Center, New York, NY, USA. ${ }^{4}$ Mayo Clinic, Rochester, MN, USA.

Received: 26 February 2019 Accepted: 26 May 2019

Published online: 08 June 2019

\section{References}

1. Kurtz SM, Ong KL, Lau E, Bozic KJ. Impact of the economic downturn on total joint replacement demand in the United States: updated projections to 2021. J Bone Jt Surg Am. Exponent, Inc., 3440 Market Street, Suite 600, Philadelphia, PA 19104. E-mail address for S.M. Kurtz: skurtz@exponent.com Exponent, Inc., 3440 Market Street, Suite 600, Philadelphia, PA 19104. E-mail address for S.M. Kurtz: skurtz@exponent.com Exponent, I. 2014;96:624-30.

2. Hamidi MJB. Changing epidemiology of the American population. Clin Geriatr Med. 2019:35:1-12.

3. Flegal KM, Kruszon-Moran D, Carroll MD, Fryar CD, Ogden CL. Trends in obesity among adults in the United States, 2005 to 2014. JAMA. 2016;315: 2284-91.

4. Merchant FM, Dickert NW, Howard DH. Mandatory shared decision making by the Centers for Medicare \& Medicaid Services for cardiovascular procedures and other tests. JAMA. 2018;320:641-2.

5. American Academy of Orthopaedic Surgeons. Shared physician-patient responsibilities. Position statement 1182. 2011. Available from: https://www. aaos.org/About/Statements/Position/

6. Wieringa $T H$, Kunneman M, Rodriguez-Gutierrez R, Montori VM, de Wit M, Smets EMA, et al. A systematic review of decision aids that facilitate elements of shared decision-making in chronic illnesses: a review protocol. Syst Rev. 2017;6:155.

7. Elwyn G, Durand MA, Song J, Aarts J, Barr PJ, Berger Z, et al. A three-talk model for shared decision making: multistage consultation process. BMJ. 2017;359:j4891. 
8. Clayman ML, Bylund CL, Chewning B, Makoul G. The impact of patient participation in health decisions within medical encounters: a systematic review. Med Decis Mak. 2015;36:427-52.

9. Aubree Shay L, Lafata JE. Where is the evidence? A systematic review of shared decision making and patient outcomes. Med Decis Mak. 2015;35: 114-31.

10. Grant FC, Laupacis A, O'Connor AM, Rubens F, Robblee J. Evaluation of a decision aid for patients considering autologous blood donation before open-heart surgery. CMAJ. 2001;164:1139-44.

11. Stacey D, Légaré $F$, Lewis K, Barry MJ, Bennett $C L$, Eden KB, et al. Decision aids for people facing health treatment or screening decisions. Cochrane Database Syst Rev. 2017.

12. Shamseer $L$, Moher D, Clarke M, Ghersi D, Liberati A, Petticrew M, et al. PRISMA-P (Preferred Reporting Items for Systematic review and MetaAnalysis Protocols) 2015 checklist: recommended items to address in a systematic review protocol *. BMJ. 2015;349:97647.

13. Bozic KJ, Belkora J, Chan V, Youm J, Zhou T, Dupaix J, et al. Shared decision making in patients with osteoarthritis of the hip and knee. J Bone Jt Surg. 2013;95:1633-9.

14. Stacey D, Taljaard M, Dervin G, Tugwell P, O'Connor AM, Pomey MP, et al. Impact of patient decision aids on appropriate and timely access to hip or knee arthroplasty for osteoarthritis: a randomized controlled trial. OsteoarthritisCartilage. University of Ottawa, Faculty of Health Sciences, Canada; Ottawa Hospital Research Institute, Clinical Epidemiology Program, Canada Electronic address: dstacey@uottawaca Ottawa Hospital Research Institute, Clinical Epidemiology Program, Canada; Universi. 2016;24:99-107.

15. Landis JR, Koch GG. The measurement of observer agreement for categorical data. Biometrics. 1977;33:159-74.

16. O'connor AM. Validation of a decisional conflict scale. Med Decis Mak. 1995; 15:25-30.

17. Higgins, JPT, Sterne, JAC, Savovic, J, Page, MJ, Hrobjartsson, A, Boutron, I, Reeves, B, Eldridge $S$. A revised tool to assess risk of bias in randomized trials (RoB 2). 2018 [cited 2019 Mar 28]. Available from: https://sites.google. com/site/riskofbiastool/welcome/rob-2-0-tool

18. Viechtbauer W. Conducting meta-analyses in $\mathrm{R}$ with the metafor package. J Stat Softw. 2010;36:1-48.

19. R Development Core Team. R: a language and environment for statistical computing. R Found. Stat. Comput. 2019. Available from: http://www.rproject.org/

20. Peters JL, Sutton AJ, Jones DR, Abrams KR, Rushton L. Contour-enhanced meta-analysis funnel plots help distinguish publication bias from other causes of asymmetry. J Clin Epidemiol. 2008;61:991-6.

21. de Jesus C, Stacey D, Dervin GF. Evaluation of a patient decision aid for unicompartmental or total knee arthroplasty for medial knee osteoarthritis. J Arthroplast. 2017;32:3340-4.

22. Ibrahim SA, Blum M, Lee GC, Mooar P, Medvedeva E, Collier A, et al. Effect of a decision aid on access to total knee replacement for black patients with osteoarthritis of the knee a randomized clinical trial. JAMA Surg. 2017; 152:e164225.

23. Schunemann, H, Brozek, J, Guyatt, G, Oxman A. GRADE handbook. 2013 [cited 2019 Mar 28]. Available from: https://gdt.gradepro.org/app/ handbook/handbook.html

24. GRADEpro GDT [Internet]. [cited 2019 Mar 28]. Available from: https:// gradepro.org/

25. de Achaval S, Fraenkel L, Volk RJ, Vanessa Cox MES-A, De Achaval S, Fraenkel L, Volk RJ, Cox V, Suarez-Almazor ME. Impact of educational and patient decision aids on decisional conflict associated with total knee arthroplasty. Arthiritis Care Res. 2012;64:229-37.

26. Stacey D, Hawker G, Dervin G, Tugwell P, Boland L, Pomey MP, et al. Decision aid for patients considering total knee arthroplasty with preference report for surgeons: a pilot randomized controlled trial. BMC Musculoskelet Disord. 2014;15:54.

27. Shue J, Karia RJ, Cardone D, Samuels J, Shah M, Slover JD. A randomized controlled trial of two distinct shared decision-making aids for hip and knee osteoarthritis in an ethnically diverse patient population. Value Heal. 2016; 19:487-93.

28. Vina ER, Richardson D, Medvedeva E, Kent Kwoh C, Collier A, Ibrahim SA Does a patient-centered educational intervention affect African-American access to knee replacement? A randomized trial. Clin Orthop Relat Res. 2016;474:1755-64.
29. Bunzli S, Nelson E, Scott A, French S, Choong P, Dowsey M. Barriers and facilitators to orthopaedic surgeons' uptake of decision aids for total knee arthroplasty: a qualitative study. BMJ Open. 2017;7:1-16.

30. Agoritsas T, Heen AF, Brandt L, Alonso-Coello P, Kristiansen A, Akl EA, et al: Decision aids that really promote shared decision making: the pace quickens. BMJ. 2015;350:g7624.

31. Schaffner KF. Clinical trials and causation: Bayesian perspectives. Stat Med. 1993;12:1477-94.

\section{Publisher's Note}

Springer Nature remains neutral with regard to jurisdictional claims in published maps and institutional affiliations.
Ready to submit your research? Choose BMC and benefit from:

- fast, convenient online submission

- thorough peer review by experienced researchers in your field

- rapid publication on acceptance

- support for research data, including large and complex data types

- gold Open Access which fosters wider collaboration and increased citations

- maximum visibility for your research: over $100 \mathrm{M}$ website views per year

At BMC, research is always in progress.

Learn more biomedcentral.com/submissions 